Cahiers de recherches médiévales

Journal of medieval studies

15 | 2008

La Tentation du parodique dans la littérature médiévale

\title{
Courtois d'Arras et le Jeu de la Feuillée
}

De l'imitation créatrice à la parodie subversive

Jean Dufournet

\section{(2) OpenEdition}

Journals

Édition électronique

URL : https://journals.openedition.org/crm/5573

DOI : $10.4000 / \mathrm{crm} .5573$

ISSN : 1955-2424

Éditeur

Honoré Champion

Édition imprimée

Date de publication : 20 juin 2008

Pagination : 45-58

ISSN : 1272-9752

Référence électronique

Jean Dufournet, «Courtois d'Arras et le Jeu de la Feuillée », Cahiers de recherches médiévales [En ligne],

15 | 2008, mis en ligne le 20 juin 2011, consulté le 15 décembre 2022. URL : http://

journals.openedition.org/crm/5573; DOI : https://doi.org/10.4000/crm.5573 


\title{
rin
}

\author{
Courtois d'Arras et le Jeu de la Feuillée: \\ de l'imitation créatrice à la parodie subversive
}

\begin{abstract}
The thirteenth-century secular theatre is particularly interesting insofar as it is a true literary laboratory based on texts with all kinds of plays whose purpose is not always easy to grasp. The introductory masterpiece of this tradition is Jean Bodel's Jeu de saint Nicolas, written and performed around 1200. In its wake, two works appeared which represent two generations of plays within the thirteenth century: one, Courtois d'Arras, dating from the first quarter of the century, is a subtle variation on the parable of the prodigal son (Luke 15:11-32); in the other, Jeu de la Feuillée, performed on June 3, 1276, Adam de la Halle incorporates his own persona and his entire work, which he uses as subtext. But the two plays have most likely been modeled on the Jeu de St Nicolas, to which they make many allusions. From the start it should be pointed out that the Jeu de la Feuillée is based on the Jeu de St Nicolas, reworked and completed with Courtois d'Arras. In addition, if the latter text is given over to an up-dating of the prodigal son parable and a humanizing of religion as well as to an ironic use of courtoisie, the parody becomes generalized in the Jeu de la Feuillée and ends up totally subverting and destroying all hopes and dreams in a universe where God no longer appears, but where the all-powerful Fortune reigns in irrational and arbitrary triumph. The wheel of Fortune, which never stops turning, has influence over the powerful men of Arras, over the fairies (fées) and finally, more generally, over all the other characters, who come back one by one on the turning wheel which becomes more and more frenetic, each turn bringing a character to the front of the stage, to point out his decline and defeat.
\end{abstract}

Résumé : Le théâtre profane du XIII siècle est pour la littérature un véritable laboratoire. Le chef-d'œuvre inaugural de cette nouvelle tradition est le Jeu de saint Nicolas de Jean Bodel, représenté à Arras vers 1200. Dans son sillage on dénombre deux auvres qui sont caractéristiques de deux générations du XIII siècle. La première, Courtois d'Arras, datant du premier quart de ce siècle, est une subtile variation sur la parabole du fils prodigue. Dans la seconde, le Jeu de la Feuillée, représentée le 3 juin 1276, l'auteur, Adam de la Halle, joue avec son propre personnage et avec l'ensemble de son æuvre qui lui sert d'hypotexte. Mais les deux pièces ont à coup sûr comme modèle le Jeu de saint Nicolas auquel elles font de multiples allusions. Il faut préciser que la Feuillée se situe par rapport à un Jeu de saint Nicolas revu et complété par Courtois d'Arras. D'autre part, si ce texte-ci se livre tout autant à une actualisation de la parabole et à une humanisation de la religion qu'à une utilisation ironique du Conte du Graal et de la courtoisie, la parodie se généralise dans la Feuillée et aboutit à une subversion totale qui détruit tous les espoirs et les rêves dans un univers où Dieu n'apparaît plus, tandis que la toute-puissante roue de Fortune règne et triomphe de façon totalement arbitraire : ne cessant de tourner, elle exerce son influence néfaste sur les puissants d'Arras, sur les fées, sur chacun des personnages de la pièce dont elle détermine le déclin et la défaite.

Le théâtre profane du XIII ${ }^{\mathrm{e}}$ siècle est particulièrement intéressant dans la mesure où c'est un véritable laboratoire littéraire qui se fonde sur toutes sortes de

Cahiers de Recherches Médiévales, 15, 2008 
jeux intertextuels dont il n'est pas toujours facile de saisir la finalité. Le chefd'œuvre inaugural de cette tradition est le Jeu de saint Nicolas de Jean Bodel, écrit et joué vers 1200. Dans son sillage se sont inscrites deux oeuvres, représentatives de deux générations du XIII' siècle : l'une, Courtois d'Arras, datant du premier quart de ce siècle, est une subtile variation sur la parabole de l'Enfant prodigue (Luc, XV, 1132); dans l'autre, le Jeu de la Feuillée, représenté le 3 juin 1276, Adam de la Halle joue avec son propre personnage et avec l'ensemble de son oeuvre, qui lui sert d'hypotexte. Mais les deux pièces ont pris, à coup sûr, comme modèle le Jeu de saint Nicolas auquel elles font de multiples allusions. Il faut tout de suite préciser que le Jeu de la Feuillée se situe par rapport à un Jeu de saint Nicolas, revu et complété par Courtois d'Arras. D'autre part, si ce texte-ci se livre tout autant à une actualisation de la parabole et à une humanisation de la religion qu'à une utilisation ironique de la courtoisie, la parodie se généralise dans le Jeu de la Feuillée et aboutit à une subversion totale. Ce sont deux étapes de la désacralisation du monde. ${ }^{1}$

Pour la parodie on se référera à la bonne mise au point de Claude Lachet dans le numéro de L'École des Lettres ${ }^{2}$ consacré à Aucassin et Nicolette :

«... le parodiste ne s'attache pas uniquement à l'expression de son ou de ses devanciers ; il s'intéresse à l'écriture, certes, mais aussi aux motifs, aux situations, au héros, à tout l'univers imaginaire de son ou ses modèles. Par conséquent, la parodie est une transformation ludique par laquelle un écrivain transpose le sujet, les thèmes, les idées, les personnages, la signification et l'esthétique d'un texte ou d'un ensemble de textes, dans l'intention de provoquer le rire ou le sourire d'un public dont il recherche la connivence. Elle ressortit à une double opération d'emprunt et de distanciation comique et tient dans cette oscillation constante entre la conformité et l'écart. Elle nécessite deux principes essentiels :

- la présence d'un référent (textes de base, appelée hypotextes par Gérard Genette) que l'auteur démarque et que le destinataire s'efforce de retrouver à partir du texte parodiant (nommé hypertexte par Gérard Genette);

- un jeu permanent de concordances et de dissemblances entre le parodiant et le parodié. »

I

L'auteur de Courtois d'Arras - qui a emprunté à Jean Bodel, outre le motif de la taverne qu'il a enrichi et opposé à la campagne, le personnage du prud'homme, le couple du tavernier et de son valet, les quatrains d'alexandrins - a multiplié les allusions à son modèle afin que l'auditeur puisse comparer l'un à l'autre; ces

\footnotetext{
${ }^{1}$ Nous citerons les trois oeuvres dont il sera question d'après nos éditions bilingues publiées par Flammarion dans la collection G-F : Jean Bodel, Le Jeu de saint Nicolas, 2005 ; Courtois d'Arras, 1995 ; Adam de la Halle, Le Jeu de la Feuillée, $2^{\mathrm{e}}$ éd., 1989.

${ }^{2}$ 2000-2001, 15 janvier 2001, 92e année, «La Parodie », p. 127-128. Sur la parodie, on pourra lire: Cl. Abastado, "Situation de la parodie», Cahiers du XX siècle, 6, La Parodie, Paris, Klincksieck, 1976, p.9-37; G. Genette, Palimpsestes. La littérature au second degré, Paris, Éditions du Seuil, 1982 ; G Idt, «La parodie : rhétorique ou lecture ?», Le Discours et le sujet, Nanterre, 1972-1973, p. 128-173 ; Cl. Lachet, La Prise d'Orange ou la parodie courtoise d'un genre, Paris, Champion, 1986 ; D. Sangsue, La Parodie, Paris, Hachette, 1994.
} 
rencontres sont si nombreuses qu'on a pu penser que la pièce serait de Jean Bodel. En voici quelques exemples. La fameuse tirade de Raoulet sur le vin (Saint Nicolas, v. 642-650) ${ }^{3}$ est reprise, morcelée en plusieurs passages par Courtois et l'aubergiste :

COURTOIS : Et quant fu cis vins aforez?

L'HOTE : Hui main fu perciez et forez. (127-128)

L'HOTE : Lequet, trai lui a plain tonnel

tout pur, foi que vous m'i devez. (146-147)

Pourette parle d'un vin «Cler et net et sade et bevant» (v. 210). Courtois, de son côté, rêve d'un vin «sor lie» (v. 99). L'auteur se plaît à enchérir sur son prédécesseur dont le vin était « Rampant comme escuireus en bos » (v. 645) et dont l'un des personnages dit: «Vois comme il fet le velouset» (v. 721). Celui de Courtois d'Arras «fet le lïoncel» (v. 206) : jouant sur les armes d'Auxerre, d'azur au lion d'or rampant, c'est-à-dire grimpant, debout, il présente ce vin comme généreux, corsé, tonique. L'auteur de Courtois d'Arras marche sur les traces de son prédécesseur quand il est question d' «essaier le vin», de boire un «lot», quatre pintes, environ quatre litres, d' « escot», de dettes et de gages. Si Jean Bodel se livre à des variations sur la cape de Cliquet $^{5}$ qui doit la laisser aux mains de l'aubergiste, tout en partant avec un sac de ce dernier, le héros de Courtois d'Arras s'en va avec un « sorcotelet viez » (v. 414).

De son côté, Adam de la Halle a enchéri sur les pratiques de son prédécesseur, d'abord en reprenant certains vers du Jeu de saint Nicolas, tels quels ou plus ou moins modifiés. Par exemple:

\footnotetext{
Jeu de saint Nicolas

Quia! Kia!

Chil deriere vienent du mains !

(1083-1084)

A cui iés tu?

- Je sui au roy

Si port son seel et son brief.
}

(266-267)

\author{
Jeu de la Feuillée \\ Qu'i a ? K'i a? K'i a ? K'i a ? \\ Or puis seur chou estre escoliers.
}
A cui iés tu, di, barbustin?
- Qui ? Jou?
- Voire.
- Au roy Hellekin

Qui chi m'a tramis en mesage.

\footnotetext{
${ }^{3}$ Éd. cit, p. 100-101: Le vin aforé de nouvel,/A plain lot et a plain tonnel,/Sade, bevant et plain et gros,/Rampant comme escuireus en bos,/Sans nul mors de pourri ne d'aigre,/Seur lie, court et sec et maigre,/Cler con larme de pecheour,/Croupant seur langue a lecheour:/Autre gent n'en doivent gouster! «Le vin fraîchement mis en perce, à bonne mesure et sans mélange, gouleyant, coulant, ample, corsé, grimpant comme écureuil au bois, sans aucune trace de moisi ni d'acidité, sur lie, direct, sec et léger, limpide comme une larme de pécheur, s'attardant sur la langue du gourmet : les autres gens ne doivent pas y goûter. »"

${ }^{4}$ Sur l'expression seur lie, voir notre commentaire dans notre éd. du Jeu de saint Nicolas, p. 205, note du vers 647.

${ }^{5}$ Voir notre éd., v. 665-666, 743-744, 759, 815-820, 919-920, et surtout 1319-1330.
} 
Or cha! Rasoir, venés seoir, S'arés de no commenchement.
Je n'en conterai point a ti :

Ains sera de commenchement. ${ }^{6}$

$(897-898)$

Adam de la Halle a voulu aussi établir des liens, d'une pièce à l'autre, entre certains personnages. Ainsi en est-il de l'aubergiste. Il est appelé « tavernier» par le rubricateur et « oste » par les personnages du Jeu de saint Nicolas; il est « li Ostes» dans le Jeu de la Feuillée où il est, en outre, dénommé, au vers 882, Raoul Le Waidier, qui est le nom d'un Arrageois mort en 1311, et deux fois, aux vers 904 et 928, Rauelet (Raoulet), le crieur de vins de Jean Bodel. Il vend son vin « au ban de le vile», au tarif officiel (Jeu de saint Nicolas, 258), "par eskievins » (Jeu de la Feuillée, 910); c'est, dans les deux cas, du vin d'Auxerre accompagné de harengs. D'autre part, deux messagers, appelés « sire courlieu » dans les deux pièces, relient la taverne et Arras à un autre monde (sarrasin d'un côté, et féerique de l'autre). Auberon de Bodel, derrière qui se profile le nain royal d'Huon de Bordeaux, est le modèle du Croquesot d'Adam de la Halle qui a poursuivi le travail de démythification de son prédécesseur qui faisait pénétrer Auberon dans la taverne où il buvait et jouait: Croquesot, à qui les fées offrent un verre avant de partir (v. 835), est un «barbustin », un gnome ventru et barbu, aux cheveux hérissés.

Çà et là, Adam reprend des rimes. Tantôt, dans un même éloge du vin, il complique le jeu par une rime homonymique très riche :

Jeu de saint Nicolas

Tenés, Rasoir, par uns couvens Que ne tenistes tel auwen.

$(751-752)^{7}$
Jeu de la Feuillée

Tel ne boit on mie en couvent ; Et si vous ai bien couvent Qu'auen ne vint mie d'Aucheure.

Tantôt il développe sur deux répliques l'opposition de l'écorce et du corps qui se substitue au cœur :

Jeu de saint Nicolas

CIL DU SEC ARBRE :

Sains Nicolais, c'est maugré mien
Jeu de la Feuillée

LI OSTES : Ou vous me lairés cha che froc. Le cors arés et jou l'escorche.

\footnotetext{
${ }^{6}$ Traduction des citations de Jean Bodel : «Merde de merde! Ces dés sont faits pour donner le moins !»; « À qui es-tu? - Je suis au roi dont je porte le sceau et le message »; «Eh bien! Rasoir, venez vous asseoir, vous serez de notre première tournée. » Traduction des citations d'Adam de la Halle: «Qu'est-ce qu'il y a ? Qu'est-ce qu'il y a ? Il m'est facile dans ces conditions d'être étudiant! »; «Dis-moi, barbichu, au service de qui es-tu ?- Qui ? Moi ? Oui, toi - Je sers le roi Hellequin qui m'a envoyé ici porter un message.»; «Je ne te la compterai pas, c'est seulement pour commencer. » Pour d'autres exemples, voir notre livre Sur le Jeu de la Feuillée, Paris, SEDES, 1977, p. 74-75.

${ }^{7}$ Traductions: «Tenez, Rasoir, je vous parie que vous n'en avez pas bu de tel cette année. »; «On n'en boit pas de tel dans les couvents. Et je vous le garantis : ce n'est pas cette année qu'il est venu d'Auxerre. »
} 
Que je vous aoure, et par forche. De moi n'arés vous fors l'escorche.

$(1507-1509)^{8}$
LI MOINES :

Ostes, me ferés vous dont forche?

Jean Bodel avait habilement joué avec les mètres comme il l'avait fait avec les motifs et les avant-textes. Adam de la Halle, qui privilégie, comme Jean Bodel, les octosyllabes à rimes plates, s'est borné à lui emprunter les quatrains d'alexandrins monorimes au début du Jeu de la Feuillée (v. 1-12) et les sixains d'octosyllabes en $a a b c c b$ aux v. 33-182 (portrait de sa femme Maroie), 837-872 (fin de la féerie) et 1094-1099 (fin du Jeu). Ainsi souligne-t-il l'importance de ces passages pour l'interprétation de la pièce.

Après avoir accumulé tous ces indices par une volonté d'émulation créatrice et, pour une part, de parodie, Adam termine le Jeu par une mention de l'église SaintNicolas :

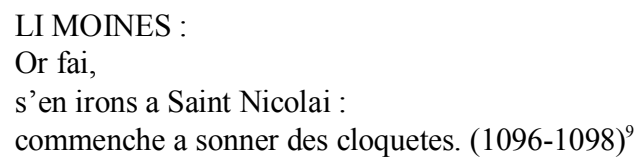

Cette signature finale évoque, s'il en était encore besoin, la pièce de Jean Bodel.

Il est aussi des reprises plus subtiles que les auditeurs avertis ne manquaient pas de saisir. Tantôt Adam de la Halle se contente d'allusions à des scènes bien connues de Jean Bodel qu'il n'a pas voulu récrire, faute de pouvoir rivaliser avec son modèle, mais qui sont à l'arrière-plan de sa pièce, comme les parties de dés ou les additions de l'aubergiste. Tantôt il prend appui sur un vers de son prédécesseur (v. 841): «se Dieus te doinst le tous», pour développer un jeu de mots plus compliqué, quand le père d'Adam, au vers 198, affirme: «Je sui uns vieus hom plains de tous » (1. plein de toux; 2. plein de tout ; 3. plaint de tous) ou sur une invocation du voleur Princedé, "Ai ! sains Lienars » (v. 1133), quand le médecin du Jeu de la Feuillée diagnostique la maladie de maître Henri : "Tu as le mal saint Lïenart» (v. 234), sur lequel les avis sont partagés ${ }^{10}$; mais le rapprochement avec le Jeu de saint Nicolas invite à penser que ce mal est la captivité dont l'ombre se profile sur les destins des trois voleurs, et dont souffrent les personnages du Jeu de la Feuillée, emprisonnés dans leur ville et leurs habitudes dont ils sont incapables de se délivrer.

\footnotetext{
${ }^{8}$ Traductions: L'ÉMIR DE L'ARBRE SEC : «Saint Nicolas, c'est malgré moi que je vous prie : j'y suis forcé. De moi vous n'aurez que l'écorce. »; L'AUBERGISTE : « Ou vous nous laisserez le froc que voici : vous garderez le corps et moi l'écorce. » LE MOINE : «Patron, me ferez-vous donc violence?»

${ }^{9}$ LE MOINE : « Allons, partons pour Saint Nicolas : commence à sonner tes clochettes. » Sur la ponctuation et le sens de ces vers, voir notre éd., p. 199, note des v. 1098-1099.

${ }^{10}$ Sur le mal saint Lienart, voir notre éd., p. 158-159, note du vers 234.
} 
Plus ironique, relevant de l'héroï-comique et de la parodie, est le réemploi de termes de Jean Bodel qui ressortissent à la société féodale. «Baron », appliqué aux plus importants vassaux du roi d'Afrique et à saint Nicolas, ne désigne plus dans le Jeu de la Fenillée que des maris qui, deux fois sur trois, ne sont pas maîtres chez eux. Quant au titre de «roi», s'il concerne dans le Jeu de saint Nicolas le puissant souverain sarrasin, qui n'est pas uniformément ridicule, il est revendiqué par le fou furieux dans le Jeu de la Feuillée (v. 395) ou appliqué aux tout-puissants favoris du comte d'Artois (v. 793), ou à Hellequin le roi de féerie qui est lui aussi suivi de sa «maisnie» et qui perd beaucoup de son prestige dans l'évocation d'une scène burlesque: caché dans un nuage de poussière, ne fait-il pas un croc-en-jambe au cheval de Robert Sommeillon (v. 737-741) ? Enfin, le «prince» n'est plus pour Adam que le «prince du pui», et le dervé affirme: «Je sui miex prinches qu'il ne soit» (v. 407).

\section{II}

Dans cet entrelacs de citations du Jeu de saint Nicolas dont l'ensemble constitue un réseau dense et suggestif, les deux successeurs ont introduit des transformations qui ne relèvent pas toutes de la parodie.

Le poète de Courtois d'Arras s'est livré à une triple opération, se plaisant d'abord, sur les brisées de Jean Bodel, à enrichir la scène de la taverne qui occupe la moitié de la pièce. Ce n'est plus une simple taverne, mais une auberge qui fait hôtel et où l'on peut boire, manger, coucher, rencontrer des femmes, obtenir du crédit merveilleux paradis terrestre où tout est beau et abondant : jardin verdoyant et fleuri, parfums, bon vin, chère plantureuse, belles chambres, crédit illimité, avenantes hôtesses. Mais, comme pour Jean Bodel, c'est le lieu trompeur du Mal, où il n'y a pas plus d'amitié et d'amour que de crédit, et où tout se paie, très cher et vite. C'est le théâtre de la ruse et de la tromperie, tant du patron et de son valet que des deux femmes, Pourette et Manchevaire, que l'auteur de Courtois d'Arras a introduites dans la pièce au point de consacrer à la première plus de cent-dix vers, et c'est son principal apport. Si Jean Bodel opposait la taverne et la cour du roi sarrasin, Courtois d'Arras oppose la campagne de la maison du père et du travail agricole chez le prud'homme à la taverne au cœur de la ville, et les deux contrées symbolisent des voies antithétiques. Courtois d'Arras grossit certains traits esquissés par Jean Bodel, mais c'est de la caricature plutôt que de la parodie, par exemple quand l'aubergiste de cette pièce vend son vin à six deniers le lot, soit le double du prix pratiqué par l'aubergiste du Jeu de saint Nicolas. Le lot contenant quatre pintes, la pinte vaut un denier et demi, alors qu'elle ne coûte qu'une maille et demie dans la pièce de Jean Bodel. ${ }^{11}$

En second lieu, l'auteur actualise la parabole de l'Enfant prodigue, qui servait peut-être de prologue à la pièce, comme le texte hagiographique consacré à saint Nicolas ouvrait le jeu de Jean Bodel. S'il reprend la trame du texte évangélique, il

\footnotetext{
${ }^{11}$ Sur les rapports de Courtois d'Arras avec le Jeu de saint Nicolas, voir notre article "Courtois d'Arras ou le triple héritage », Revue des langues romanes, 95, 1991, Naissances du théâtre français (XII - XIII ${ }^{e}$ siècles), p. 84-99.
} 
développe la débauche du héros, résumée par une phrase dans l'Évangile. «L'habitant de la contrée», qui l'emploie comme porcher, intervient personnellement : ses paroles sur Dieu, ou sur Fortune selon les manuscrits, absentes de la parabole, donnent à la pièce une autre dimension. L'opposition du père et du fils perd son caractère purement symbolique pour devenir profondément humaine. Le manuscrit $B$ ajoute même le personnage de la fille, antithétique de celui du frère aîné. Constamment, l'auteur oriente son hypotexte, jusqu'à s'éloigner de lui et le détourner de son sens. Par exemple, à la fin du jeu, au contraire de la parabole, le père semble ne pas reconnaître son fils. Les épreuves ont-elles rendu celui-ci à ce point méconnaissable, ou bien est-ce qu'il n'a pas encore accédé au repentir, se bornant à des regrets? Ou encore (plutôt) le père fait-il semblant de ne pas le reconnaître afin que Courtois, s'humiliant, confesse publiquement sa faute et retrouve sa figure humaine qu'il avait perdue par la débauche? L'on peut aller plus loin, au delà du texte évangélique, et déceler que, par l'importance accordée à la taverne, image de l'enfer, la pièce insiste sur le choix d'un Courtois responsable de sa vie et de sa mort, et aussi que le père porte une lourde responsabilité : n'a-t-il pas donné à son fils, en l'appelant Courtois, des modèles auxquels celui-ci ne pouvait se conformer et auxquels il était dangereux qu'il voulût se conformer ? N'a-t-il pas donné à ses deux fils une éducation qui a provoqué des récriminations, des revendications, une réelle difficulté à vivre en restant à sa place ? ${ }^{12}$

Tous ces éléments, qui sont pour une large part sous-jacents, constituent des ajouts qui prolongent le texte-source, et même le mettent en question, sans qu'il y ait jamais parodie. Il n'en va pas de même avec l'introduction, dans le long épisode de la taverne, des deux femmes qui permet à l'auteur de retrouver la tonalité des fabliaux. Pourette mène la ruse, dirige les opérations, écoutée et redoutée, aidée de sa compagne et complice Manchevaire. Elle manifeste une allégresse trompeuse, tant son jeu paraît naturel lorsqu'elle simule l'amour et la générosité. Elle s'acharne sur sa victime, qu'elle pousse à boire, se plaisant au double sens, lui révélant à demimot la vérité; pour bien vivre, il faut «engignier », user de ruse, et bien jouer : le denier est un bon camarade, le seul ami à cultiver (v. 311). Il se peut qu'elle trompe aussi l'aubergiste ${ }^{13}$.

Mais surtout, grâce à cet ajout, l'auteur de Courtois d'Arras peut se livrer avec délices à une parodie de la courtoisie, à commencer par le nom de son héros, Courtois, paysan mal dégrossi, qui se croit initié aux belles manières, joue au personnage raffiné, se prend pour Gauvain (v. 247) et qui, selon Pourette, « fet le cortois vilain » (v. 248). Il parle et agit en paysan parvenu. Il méprise le travail des champs, la nourriture des rustres; il passe son temps à jouer et se flatte de ne pas thésauriser. Son idéal est de vivre dans l'opulence et le raffinement. Il veut changer d'horizon: "Molt voit qui va par le païs » (v. 117). Courtois est la caricature du chevalier errant. Il appelle Pourette « Ma demoiselle, ma douce amie», et rappelle qu'il est au service des dames («quar fame ne haï je onques », v. 157), devant qui il s'efface. Il pratique le secret dans les affaires d'amour. Il trouve de la noblesse au jardin qui n'est qu'un « courtil », un jardin potager : «Comme il i fet bel et gentil» (v. 280). Il entend respecter les nobles coutumes en se lavant la bouche et le visage :

${ }^{12}$ Ibid., p. 100-106.

${ }^{13}$ Ibid., p. 94-97. 
Il est généreux avec ostentation, proclamant : «Certes ja n’en ferai l'aver» (v. 294). Il se soumet même au rite du don contraignant :

Or pues dire quanques tu viex,

quar je l'otroi sanz contredire. (v. 302-303)

Jouant son rôle jusqu'au bout, il affirme, comme un poète courtois, qu'il reste « en ostage » chez l'aubergiste (v. 351).

Pourette, qui a compris à qui elle a affaire, abonde dans son sens : elle lui donne du «sire damoisieus » (v. 150) et du «biaus dous amis» (v. 158); elle lui parle en termes flatteurs de ses yeux (comme s'il s'agissait de Perceval), de ses belles dents et de sa bouche (v. 149-152). Très vite, elle lui accorde, avec ironie, qu'il ne dépare pas leur compagnie : «Ja sanblez vous de nostre gent» (v. 153) et qu'il n'a rien d'un vilain : son cœur lui fait sentir qu'il est courtois et intelligent (v. 162-164). Elle souhaite d'avoir « un si bel ami », qu'elle hausse au niveau des rois et des comtes, et qui peut tout obtenir sans rien faire (v. 165-169). Plaisante allusion, in fine, au métier de Pourette, le plus vieux du monde. Et Manchevaire d'enchérir : ils formeront un beau couple, car Pourette aime Courtois "par amors ", et c'est une «dame avenant et bele et cointe,/bien renvoisie et bien repointe» (v. 191-192). Le dernier adjectif, qui signifie «habile», fait éclater l'aveuglement du paysan. Pourette continue à le griser par la répétition du mot «amour», tout en le faisant boire (v. 214-221). Après le retour de Courtois, qui avait été éloigné pour que Pourette révèle ses intentions et ses véritables sentiments, la comédie courtoise se poursuit: la séductrice demande eau chaude et serviette pour que «cis biaus meschins » puisse laver «sa bele bouche et son biaus vis» (v. 282-283c) et du vin : «l'en doit boivre aprés le laver» (v. 293). Pour finir, elle souhaite lui demander conseil (v. 293).

Ainsi découvre-t-on en filigrane une fine contestation de la courtoisie. La dame, la douce amie, n'est qu'une prostituée «bien gaagnant et bien repointe»; sa suivante, qui joue le rôle de Lunete auprès de Laudine dans Le Chevalier au lion, est une maquerelle vieillie, portée sur la boisson. Le vin, consommé en grande quantité, a remplacé le philtre de Tristan et Iseut auquel on peut voir une allusion dans les vers 220-221: « et j'en apele le bevrage / de ceste amor qui s’i afruite» («Cette boisson est le témoignage de cet amour qui est si profitable»), ainsi que dans le hanap d'argent qu'on trouve dans le Tristan d'Oxford ${ }^{14}$. Ce chevalier servant a pour arme une bourse bien enflée, appelée «loquerele» (v. 78), « massue»; or celle-ci est l'arme des fous, des géants et des hommes sauvages. La «raverdie» est un tapis

${ }^{14}$ Tristan et Iseut. Les poèmes français, la saga norroise, éd. et traduits par D. Lacroix et $\mathrm{Ph}$. Walter, Paris, Le Livre de Poche, 1989 (Lettres gothiques), p. 258, v. 649-652 : Un valet ki a mes pez sist / Levat e le costerel prist. / En un hanap d'argent versat / Le baivre ke il denz truvat, / Puis m'assist le hanap et puing / E je en bui a cel besuing. 
d'herbe sur lequel il ferait bon s'étendre pour manger du jambon et boire un plein pot de vin «sor lie». Le verger de l'amour est un «biau cortil» où le héros se rend pour «estaler», «uriner». La taverne se substitue à la chambre d'amour et à la cour; la «fin'amor» dont se réclame Pourette n'est qu'une tromperie qui, plus encore que les dés et le vin, dépouille ce Gauvain de pacotille.

Pour accentuer la parodie, que la mise en scène peut souligner, l'auteur a multiplié les antiphrases, les euphémismes pour rappeler la profession de Pourette qui, par exemple, va « en son afere, la ou nous savons no conquest » (v. 328-329), ou les doubles sens. Ainsi au vers 153, «Ja sanblez vous de nostre gent» signifie tout autant «vous semblez faire partie de la société courtoise comme nous », que «vous semblez bien être un vilain comme nous », ou encore au vers 169: aucun prince n'obtint autant de richesses et de plaisirs que Courtois aurait «sanz oevre faire», « sans rien faire» (car il sera entretenu par Pourette) ou « sans faire l'amour $»^{15}$.

\section{III}

Dans le Jeu de la Feuillée, la parodie se généralise au service d'une subversion radicale, à partir d'éléments empruntés soit au Jeu de saint Nicolas, soit à Courtois d'Arras, soit aux deux pièces. Bien entendu, Adam de la Halle n'a pas repris la trame du Jeu de saint Nicolas, qu'il a remplacée par son propre itinéraire (réel ou rêvé), mais des éléments essentiels qui lui permettaient de se livrer à une recréation en profondeur et de faire table rase d'un ensemble de clichés, de pratiques et de croyances. Il a opéré une véritable déconstruction de son modèle.

Il est présenté comme le clerc à la cape (v. 422), la cape des clercs parisiens, qui est son nouvel « abit » (v. 1), mais qui rappelle aussi la cape de Cliquet, l'un des trois voleurs de Jean Bodel, sur laquelle celui-ci plaisante en plusieurs variations, avant qu'elle ne tombe entre les mains de l'aubergiste comme gage de la dette. N'est-ce pas annoncer le destin d'Adam s'il demeure à Arras ? Il deviendra un clerc déchu qui, à force de fréquenter les tavernes, perdra jusqu'à sa cape, jusqu'aux marques extérieures de sa dignité de clerc.

D'autre part, Adam de la Halle est parti d'une suggestion de Courtois d'Arras pour mettre en scène les relations $d u$ père et $d u$ fils. Cette pièce, qui commence par un différend entre les deux hommes, se termine d'une manière qui peut sembler ambiguë. Faut-il voir, dans le silence final du héros, un indice du retour humiliant dans le giron paternel, ou bien, conformément au sens de la parabole évangélique, Courtois retrouve-t-il le modèle paternel auquel il doit se soumettre pour recouvrer son humanité ? Adam de la Halle a exploité la première possibilité : il suit son père à la taverne (v. 957-959) après qu'il a échoué à échapper à l'emprise dégradante d'Arras, et il ne prononce plus un mot. Bien plus, le poète a développé deux portraits de père, avec maître Henri et le père du « dervé ». Les deux ont en commun de se plaindre de ne rien posséder. Mais le premier, s'il ne bat pas son fils, est peint sous les plus noires couleurs : enfermé dans son égoïsme, il ne fait rien pour aider Adam; au contraire, il l'enfonce de nouveau dans la vie arrageoise en l'entraînant à la taverne. Le second, en revanche, fait son possible pour obtenir la guérison de son fils dont il supporte avec patience les excentricités et les coups ; il

${ }^{15}$ Sur tout cela, voir notre art. cit., p. $76-84$. 
essaie de le calmer et ne se met en colère que lorsque le fou se livre à des manifestations obscènes; il revient avec lui auprès des reliques de saint Acaire; enfin son désespoir le fait céder à la violence verbale et matérielle. Ce double portrait, s'il accentue la satire contre maître Henri, révèle, par le dialogue et les jeux de scène, les rapports réels des hommes entre eux, faits de violence et de haine, masqués par l'hypocrisie, riches d'injures, de disputes et de coups. ${ }^{16}$

Les trois amis d'Adam, Riquier (appelé aussi Riquece Aurri), Hane le Mercier et Guillot le Petit, reproduisent le trio des mauvais garçons du Jeu de saint Nicolas, Cliquet, Pincedé et Rasoir, qui étaient eux-mêmes une réplique de trois truands d'un fabliau du même Jean Bodel, Barat, Travers et Haimet; ils rappellent aussi les trois paysans du Jeu de Robin et Marion d'Adam de la Halle lui-même, Gautier, Baudon et Huart. Ce qui nous invite à douter de l'honnêteté, de la sobriété, de la finesse et de l'intelligence des trois compères d'Adam. Comme Jean Bodel, l'auteur du Jeu de la Feuillée a choisi des noms parlants: Riquier est à mettre en rapport avec la richesse, Hane avec la sottise et l'âne, Guillot avec la «guile», la ruse. Adam va encore plus loin que son prédécesseur, puisqu'il donne aux trois amis le nom de personnages réels, en sorte que, de ce feuilleté sémique, se dégage une complexité ambiguë, qui est celle de la vie ${ }^{17}$.

Le travail de réécriture et de déconstruction est encore plus subtil avec le personnage du moine qui joue un rôle important dans le Jeu de la Feuillée luimême, alors que le prêcheur de Jean Bodel se cantonnait à présenter l'histoire du miracle dans le prologue du Jeu de saint Nicolas ${ }^{18}$. Celui-ci parle de saint Nicolas «le confés / Qui tant biaus miracles a fais» (v. 5-6) et il relate « le haut miracle du bon saint» (v. 97) dans un panégyrique que reprend ensuite le preudome (v. 518531). Celui-là, dans le Jeu de la Feuillée, entre en scène, au vers 322, par un petit sermon patelin et plein d'onction, qui fait la réclame, à grand renfort de répétitions, d'exagération et d'amplification, de saint Acaire: ce dernier, plus efficace que tous les saints d'Irlande, guérit toutes les formes de la folie, jusqu'aux débiles les plus profonds et aux fous furieux. Mais on est fixé rapidement sur la puissance des reliques, et sur la sincérité du moine, puisque le dervé continue à manifester sa démence: il essaie de monter sur le dos de son père, il le frappe, si bien que le moine conseille au père de remmener son fils (v. 544-551), constatant: "Aussi ne fait fors rabaches » ('du tapage'). Les reliques sont tout à fait inefficaces.

Ensuite, confronté à la féerie, il accepte de céder la place aux fées, tout en demandant à assister à la scène (v. 568-573). Mais il sombre bientôt dans le sommeil, qui symbolise la léthargie intellectuelle et morale. Plus tard, après avoir accepté lui aussi d'aller à la taverne, il s'endort de nouveau (v. 963), et les autres en profitent pour le tromper et lui imputer une dette de douze sous.

Pour payer sa dette, plutôt que d'abandonner son froc comme le lui propose l'aubergiste, il laisse en gages ses reliques, et tout le monde de trouver qu'elles sont en d'aussi bonnes mains (v. 1012-1018). Il devient grossier (il emploie deux fois le

\footnotetext{
${ }^{16}$ Pour des compléments, voir notre livre Adam de la Halle à la recherche de lui-même ou le Jeu dramatique de la Feuillée, Paris, SEDES, 1974, p. 336-340.

${ }^{17}$ Sur ces personnages, voir Sur le Jeu de la Feuillée, Paris, SEDES, 1977, p. 119-124.

${ }^{18}$ Nous pensons avec un certain nombre de critiques que ce prologue est bien de Jean Bodel. Voir notre éd. cit.,p, 188, note du vers 1.
} 
verbe « conchier »), et sa colère éclate quand il voit revenir le fou, alors qu'il n'a plus ses reliques. Loin de plaindre le père du dervé qui se désespère et que menace la mendicité, il l'accable : «En vérité, vous l'avez bien mérité. Pourquoi le ramenezvous ici ?» (v. 1047-1048). Il maudit l'aubergiste et sa taverne; il s'en va, car il ne gagne plus d'argent: il ne reste autour de lui que «des gamines, des enfants et de la valetaille». Ce qui montre bien le cas que l'on fait de sa personne et de ses reliques ${ }^{19}$.

Il faut insister sur deux traits proprement parodiques. D'une part, comme les voleurs du Jeu de saint Nicolas s'endorment d'un profond sommeil durant lequel saint Nicolas intervient pour les contraindre à restituer le trésor du roi païen, de même le moine du Jeu de la Feuillée s'endort sans qu'il ait de vision, et le tavernier en profite pour lui attribuer la somme engagée. D'autre part, si le jeu de Jean Bodel vise à exalter la puissance de saint Nicolas qui, intervenant directement et convainquant les trois voleurs, sauve «le preudome» et convertit les païens par un vrai miracle qui double le trésor royal, en revanche les reliques de saint Acaire, qui sont peut-être fausses, sont inefficaces : le monde du Jeu de la Feuillée est un monde sans miracle ${ }^{20}$

Dans le Jeu de saint Nicolas, le roi d'Afrique interroge son dieu Tervagan dans une scène cocasse moins par l'ambiguïté de la réponse que par le jeu des mimiques contrastées et par la défiance du sénéchal à l'égard de son maître (v. 175200). Dans le Jeu de la Feuillée, maître Henri et Dame Douche consultent le médecin qui se dit «fisicien», c'est-à-dire tenant de la médecine savante: il commence par fonder son diagnostic sur l'examen des urines. Mais, dès qu'on conteste son jugement, cet homme si sûr de lui, qui annonce les médecins de Molière, recourt à l'onychomancie, à l'examen du pouce d'un jeune garçon (v. 258$264)^{21}$. Finalement, après la féerie, quand il met en garde les Arrageois contre les excès de l'alcool et de la bonne chère, et que Guillot dénie toute valeur à ses propos, il accepte aussitôt de s'attabler et de boire (v. 1001-1011). Si, dans les deux pièces, la colère du consultant, ici le Roi d'Afrique, là, Dame Douche, éclate dès que la vérité est révélée, le comique vient surtout de l'écart entre les objets des deux consultations : dans la première, il s'agit du grand affrontement entre païens et chrétiens; dans la seconde, qui touche au burlesque, il est question des plaisirs de la chair et des mesquineries d'Arras.

Le prud'homme joue un rôle central dans le Jeu de saint Nicolas. Vilain ordinaire, il ne participe pas au combat. Terrorisé par les Sarrasins, il a une confiance inébranlable en saint Nicolas et accepte d'être un jouet entre les mains du Tout-Puissant. Il pleure abondamment. Il s'abandonne à Dieu, conscient de sa faiblesse qui apparaît dans ses professions de foi en l'efficacité de saint Nicolas. C'est cet être démuni qui triomphe quand les chevaliers échouent, puisqu'il convertit le roi païen et sa cour. Il illustre le paradoxe chrétien de la force du faible, fort de la

\footnotetext{
${ }^{19}$ Adam de la Halle à la recherche de lui-même, op. cit., p. 320-325.

${ }^{20}$ Sur le Jeu de la Feuillée, op. cit., p. 88-89.

${ }^{21}$ La scène est bien suggérée par Adam de la Halle: on frottait d'huile ou de cire le pouce d'un jeune garçon, on exposait le pouce au soleil ou à la lumière, on nettoyait légèrement et on demandait au garçon ce qu'il voyait dans les dessins ainsi formés. Cette scène est peut-être un écho parodique de celle déjà burlesque du Jeu de saint Nicolas dans laquelle le roi païen s'engage en faisant claquer l'ongle de son pouce sur sa dent ; voir notre éd. cit., v. 198-204.
} 
puissance divine, et aussi la promesse qu'à la fidélité des hommes répondra celle de Dieu $^{22}$. L' «habitant» de la parabole prend, dans Courtois d'Arras, les traits d'un prud'homme qui appartient davantage à ce monde terrestre. Il pense qu'il faut d'abord compter sur soi pour obtenir le redressement de sa destinée. Aussi, en raison de la vigueur de Courtois, ne lui fait-il pas l'aumône, mais il lui fournit du travail. Compatissant, il l'invite à se reposer avant de commencer à travailler. C'est d'ailleurs grâce à lui que Courtois, rebuté par sa condition de porcher, reprend le chemin de la maison paternelle. Dans cette pièce, conformément à la parabole, pas de miracle éclatant ni d'apparition d'ange, comme dans le Jeu de saint Nicolas ${ }^{23}$. Or quels sont les prud'hommes dans le Jeu de la Feuillée ? Maitre Henri et le père du dervé. Le rapprochement ne peut qu'être parodique et ironique, si l'on se rappelle le portrait du premier : clerc bigame, toujours geignant, égoïste, avare, cupide, ivrogne, hypocrite, homme du refus enfermé dans le monde de l'argent, longtemps au service des échevins comme ce triple sot de Connart dans le Jeu de saint Nicolas ${ }^{24}$. De surcroît, pas de conversion dans le Jeu de la Feuillée, puisque maître Henri entraîne son fils à la taverne. Ainsi donc le prud'homme, qui était un «saint homme» dans la première pièce, devient un «homme de bien » dans la deuxième et tout juste « un brave homme » dans la troisième.

Si le Jeu de saint Nicolas reste pour une part fidèle à la chanson de geste avec l'affrontement entre chrétiens et païens, avec les pratiques étranges des Sarrasins et surtout avec le roi d'Afrique entouré de ses émirs ${ }^{25}$, Adam de la Halle, à l'exception de traces fugitives, tourne résolument le dos à l'univers épique de la croisade et de l'exaltation de la foi, qui est devenu à ses yeux archaïque et périmé, et où un ange intervient pour réconforter et encourager les chrétiens. Il le remplace par le monde courtois dans la scène centrale du banquet des fées, puis dans l'épisode du roi de féerie Hellequin. Ce monde-ci, qu'il avait déjà dénoncé, dès le début de la pièce, par le récit de son amour et le double portrait de sa femme Maroie $^{26}$, il le discrédite à son tour, puisqu'il dégénère en sorcellerie et que l'on retrouve chez les fées les tares de l'humanité, et en particulier des femmes. Ce sont de hautes dames quand on ne les connaît pas; elles apparaissent comme des êtres de raison et de beauté dont le luxe des habits souligne l'éclat. Mais très vite elles révèlent leur véritable nature de femmes bien terrestres. C'est d'abord le cas de Maglore, susceptible, jalouse, rancunière, au point de faire des dons défavorables à Adam et à Riquier, décidant de n'épargner personne. Morgue, à son tour, avec l'aide d'Arsile, ne tarde pas à faire preuve de légèreté et de maladresse en contraignant Maglore à octroyer un don aux deux Arrageois. Amoureuse, elle se trompe sur le compte de Robert Sommeillon, qu'elle finit par traiter de $«$ cacoigneur $\aleph^{27}$. Ses dons, favorables au premier abord,

\footnotetext{
${ }^{22}$ Voir notre éd., p. 33.

${ }^{23}$ Voir notre éd., p. 126.

${ }^{24}$ Ils sont appelés ainsi dans le Jeu de la Feuillée, maître Henri aux vers 230 et 235, le père du dervé aux vers 522 et 544. Voir aussi Sur le Jeu de la Feuillée, op. cit., p. 90-91.

${ }^{25}$ Voir notre article: «L'Autre sarrasin, de la Chanson de Roland au Jeu de saint Nicolas de Jean Bodel », l'Exotisme dans la poésie épique française. In Memoriam Klára Csürös, Paris, l'Harmattan, 2003, p. 45-64.

${ }^{26}$ Adam de la Halle à la recherche de lui-même, op. cit., p. 69-110.

${ }^{27}$ V. 757. Le mot, composé du préfixe péjoratif ca- et de cogner, a le double sens de «querelleur, bagarreur » et de « débauché, enclin à la bagatelle».
} 
enferment les bénéficiaires dans ce qu'ils ont déjà, leur imposent de ne pas changer. Les fées bienfaisantes se révèlent aussi néfastes que la fée malfaisante. Ce sont deux aspects différents de la même personne, Maroie. Cette scène de la féerie représente le suprême espoir du poète, un rêve de raison, de beauté, de sagesse et d'amour courtois, qui peu à peu s'effrite, au fur et à mesure que les personnages prennent vie et connaissent les tares du monde réel : erreurs, disputes, tromperie, méchancetée ${ }^{28}$. $\mathrm{Au}$ cœur même de cette scène, c'est-à-dire au centre de la pièce, la roue de Fortune marque le triomphe de l'irrationnel et de l'injustice, que les fées en soient responsables ou qu'elles-mêmes soient emportées par une puissance supérieure. Pour finir, après que Morgue a promis d'être l' «amie kiere» du peu chevaleresque Hellequin, la féerie sombre dans la sorcellerie. Les fées, entraînées par Maglore, rejoignent les vieilles femmes, parmi lesquelles Dame Douche, qui symbolise la vieillesse, la laideur, la débauche, la sorcellerie, occupe la première place. Ainsi donc le monde imaginaire n'est qu'une copie du monde réel avec ses maux et ses hantises. La merveille, qui a remplacé le miracle, s'efface à son tour.

Enfin, le thème de la folie est un fil rouge qui parcourt les trois pièces. C'est, en filigrane dans le Jeu de saint Nicolas, la folie des païens rebelles au christianisme; c'est aussi la folie sacrée des chevaliers qui acceptent de mourir pour la défense et le triomphe de leur foi. Courtois d'Arras est structuré par les deux champs sémantiques de la folie (autour de l'adjectif « fol» et de ses synonymes, de périphrases et de symboles) et de la connaissance de soi («soi reconoistre ») ${ }^{29}$. Méprisant les avertissements de son père, Courtois se dénature et opte pour le monde pervers et félon, pour le jeu, la prodigalité et la paresse. Il s'enfonce dans le vice. Sa vie et son idéal se réduisent aux fausses promesses d'une taverne, en galante compagnie, sans souci du lendemain. Dépouillé de tout par l'obscurcissement de sa raison, il prend peu à peu conscience de sa faute; mais il lui faut du temps et d'autres épreuves pour passer des regrets et des remords au repentir, et pour se reconnaître. Qu'est-ce que se reconnaître? Cette sagesse, qui s'oppose à la folie, consiste à se soumettre à la loi du père qui est celle de Dieu et représente le bien; c'est accepter ses leçons sans se rebeller, rester à sa place dans son milieu et son emploi, faire sienne la loi du travail, refuser les tentations du vin, du jeu et des femmes, ne pas être esclave de l'argent pour le dépenser ou le thésauriser. C'est reconnaître sa nature, sa condition et ses devoirs, se repentir de les avoir méconnus, rentrer en soi-même. Avec le Jeu de la Feuillée, la folie se généralise à toute la société, et elle est incurable, sans jamais déboucher sur la guérison ni sur la conversion, comme dans les deux premières pièces. Le terme générique de « sots » ne désigne pas seulement la folie violente du dervé, les excentricités de Walet qui révèle son idéal - être aussi bon ménestrel et vielleur que son père, dût-on lui couper la tête - $\mathrm{d}$ 'autres cas de dérèglement mental (Colart de Bailleul et Heuvin vont à la chasse aux papillons; l'hypernerveux Wautier-à-le Main se met en colère quand on lui rappelle son surnom de Vitulus («le veau»); le neurasthénique Jean le Queux en veut à tout le monde, et il est contraint de s'aliter), mais ce mot s'applique aussi à tous les défauts de caractère et d'intelligence, aux débauchés, aux avares, aux gloutons. La folie est partout, elle a le dernier mot, puisque le dervé contraint tout le

${ }^{28}$ Adam de la Halle à la recherche de lui-même, op. cit., p. 202-209.

${ }^{29}$ Voir notre art. cit. «Courtois d'Arras ou le triple héritage », p. 106-111. 
monde à partir, et les personnages lâchent la bride à une exubérance bouffonne et satirique $^{30}$.

Si le Jeu de saint Nicolas et Courtois d'Arras se terminent tous deux par un Te Deum et par la conversion, des Sarrasins dans un cas et du héros dans l'autre, le Jeu de la Feuillée ne connaît pas un épilogue de ce genre. Au contraire, Adam suit son père à la taverne, qui semble alors occuper tout l'espace théâtral, alors qu'elle disparaît dans les deux autres pièces. Demain sera identique à aujourd'hui : dans ce monde désacralisé, plus de miracle ni de merveille ; plus de conversion ni de progrès moral ; plus de chevalerie modèle : cet idéal n'est plus représenté que par Courtois, que Pourette traite ironiquement de Gauvain (v. 247) et par le grotesque et débauché Robert Sommeillon (Jeu de la Feuillée, v. 720-757). Généralisée, la parodie a anéanti tous les espoirs et tous les rêves dans un univers où Dieu n'apparaît plus, mais où règne, toute-puissante, Fortune, triomphe de l'irrationnel et de l'arbitraire. Sa roue qui tourne sans arrêt, agit sur les puissants d'Arras, puis sur les fées qui changent et passent de l'amour à l'hostilité (envers Robert Sommeillon) de l'indifférence à l'amour (à l'égard du roi Hellequin), de la bonté à la méchanceté de la sorcellerie. Cette roue agit plus largement sur tous les autres acteurs qui reviennent tour à tour dans une ronde de plus en plus endiablée, et, chaque fois qu'elle ramène sur le devant de la scène un personnage, c'est pour marquer sa déchéance. ${ }^{31}$

Jean Dufournet Université de la Sorbonne nouvelle - Paris

\footnotetext{
${ }^{30}$ Sur la folie dans le Jeu de la Feuillée, voir notre livre Adam de la Halle à la recherche de lui-même, op. cit., p. 297-340.

${ }^{31}$ Ibid., p. 130-139.
} 